\title{
Cyclic homologies of crossed modules of algebras
}

\author{
Guram Donadze, Nick Inassaridze, Emzar Khmaladze and Manuel Ladra*
}

\begin{abstract}
The Hochschild and (cotriple) cyclic homologies of crossed modules of (not necessarily unital) associative algebras are investigated. Wodzicki's excision theorem is extended for inclusion crossed modules in the category of crossed modules of algebras. The cyclic and cotriple cyclic homologies of crossed modules are compared in terms of a long exact homology sequence, generalising the relative cyclic homology exact sequence.
\end{abstract}

Mathematics Subject Classification (2010). 18G10, 18G50, 18G60, 16E40.

Keywords. Cyclic homology, crossed module, simplicial algebra, derived functors.

\section{Introduction}

This paper deals with Hochschild and cyclic homologies of crossed modules of associative algebras (or equivalently, simplicial algebras with the associated Moore complex of length 1).

The general concept of a crossed module originates in the work of Whitehead in the late 1940s [19]. Namely, it was introduced as an algebraic model for a pathconnected $\mathrm{CW}$-space whose homotopy groups are trivial in dimensions $>2$. There the crossed modules were crossed modules of groups and since their introduction they have played an important role in homotopy theory. For illustration we mention various classification problems for low-dimensional homotopy types and derivation of van Kampen theorem generalisations (see the survey by Brown [4]).

Crossed modules of Lie and associative algebras have also been investigated by various authors. Namely, in the works of Dedecker and Lue [6], [17] crossed modules of associative algebras have played a central role in what must be coefficients in lowdimensional non-abelian cohomology. In [3] Baues and Minian have shown that crossed modules of associative algebras can be used to represent the Hochschild cohomology. In [14] Kassel and Loday have used crossed modules of Lie algebras as computational tools in order to give an interpretation of the third relative ChevalleyEilenberg cohomology of Lie algebras.

${ }^{*}$ The authors were supported by MICINN grant MTM2009-14464-C02 (European FEDER support included) and by Xunta de Galicia grant Incite09207215PR. The first three authors were also partially supported by Volkswagen Foundation Ref.: I/84 328. 
The study of (co)homological properties of crossed modules in the category of groups has been the subject of several papers. We point out two (co)homology theories of crossed modules of groups, one introduced and investigated in the works of Baues [2] and Ellis [9] via classifying spaces, and other defined by Carrasco, Cegarra and R.-Grandjeán [5] as cotriple (co)homology. The goal of the paper of R.-Grandjeán, Ladra and Pirashvili [11] was to find a relation between these two homology theories. The motivation of our work was to extend the result of [11] to other homology theories of crossed modules of different algebraic objects.

Inspired by the recent results of [7] presenting the cyclic, periodic cyclic and negative cyclic homologies of associative algebras as cotriple derived functors (see also [10]), we construct a cotriple cyclic homology theory of crossed modules of algebras, generalising the usual cyclic homology of algebras in zero characteristic case. The main goal of this paper is to relate the cotriple cyclic homology of crossed modules with the cyclic homology of their nerves in terms of a long exact homology sequence. Another goal is to extend Wodzicki's excision theorem for inclusion crossed modules in the category of crossed modules of algebras.

1.1. Organisation. After the introductory Section 1, the paper is organized in four sections. In Section 2 we recall some necessary notions about crossed modules of associative algebras and the Hochschild and cyclic homologies of simplicial associative algebras. In Section 3 we investigate Hochschild and cyclic homologies of crossed modules of algebras. Namely, we give the five-term exact sequences relating the low dimensional Hochschild (resp. cyclic) homologies of crossed modules of algebras and their cokernel algebras (Theorem 3.2). We study the excision problem for Hochschild (resp. cyclic) homology of inclusion crossed modules of algebras (Theorem 3.4). In Section 4 we construct and study the cotriple cyclic homology theory in the category of crossed modules of associative algebras. We show that the cotriple cyclic homology of an inclusion crossed module is isomorphic to the relative cyclic homology (Proposition 4.8). In Section 5 we compare the cyclic and cotriple cyclic homology theories of crossed modules of associative algebras in terms of a long exact homology sequence (Theorem 5.1).

1.2. Notations and Conventions. We fix $\mathbb{k}$ as a ground field and write $\otimes$ for $\otimes_{\mathbb{k}}$. Vector spaces are considered over $\mathbb{k}$ and their category is denoted by Vect, while $\mathfrak{V}_{\geq 0}$ is the category of non-negatively graded complexes of vector spaces. Algebras are (non-unital) associative algebras over $\mathbb{k}$ and their category is denoted by Alg. The term free algebra means a free (non-unital) algebra over some vector space. Ideals are always two-sided. For any functor $T: \underline{C} \rightarrow$ Vect and for any simplicial object $C_{*}$ in $\underline{C}$, denote by $T\left(C_{*}\right)$ the simplicial vector space obtained by applying the functor $T$ dimension-wise to $C_{*}$. 


\section{Preliminaries}

2.1. Crossed module and its nerve. We recall the basic notions about crossed modules of algebras (cf. [8]).

Let $A$ and $R$ be two algebras. By an action of $A$ on $R$ we mean an $A$-bimodule structure on $R$ satisfying the conditions

$$
a\left(r r^{\prime}\right)=(a r) r^{\prime}, \quad(r a) r^{\prime}=r\left(a r^{\prime}\right), \quad\left(r r^{\prime}\right) a=r\left(r^{\prime} a\right)
$$

for all $a \in A, r, r^{\prime} \in R$. For example, if $R$ is an ideal of the algebra $A$, then the multiplication in $A$ yields an action of $A$ on $R$. Note that if $R$ is unital, an action of $A$ on $R$ can be just presented as an algebra homomorphism from $A$ to $R$.

Given an algebra action of $A$ on $R$, denote by $[A, R]$ the vector subspace of $R$ generated by the elements $[a, r]=a r-r a$ for $r \in R, a \in A$. Moreover, one can form the semidirect product algebra, $R \rtimes A$, with the underlying vector space $R \oplus A$ endowed with the multiplication given by

$$
(r, a)\left(r^{\prime}, a^{\prime}\right)=\left(r r^{\prime}+a r^{\prime}+r a^{\prime}, a a^{\prime}\right)
$$

for $(r, a),\left(r^{\prime}, a^{\prime}\right) \in R \rtimes A$.

A crossed module $(R, A, \rho)$ of algebras is an algebra homomorphism $\rho: R \rightarrow A$ together with an action of $A$ on $R$ such that

$$
\begin{gathered}
\rho(a r)=a \rho(r), \quad \rho(r a)=\rho(r) a, \\
\rho(r) r^{\prime}=r r^{\prime}=r \rho\left(r^{\prime}\right) \quad(\text { Peiffer identity) }
\end{gathered}
$$

holds for all $a \in A, r, r^{\prime} \in R$. We point out that the image of $\rho$ is necessarily an ideal of $A$, and that $\operatorname{Ker} \rho$, contained in the two-sided annihilator of $R$, is an $A / \rho R$-bimodule.

The concept of a crossed module of algebras generalises simultaneously the concepts of ideal and bimodule. In fact, a common instance of a crossed module of algebras is that of an algebra $A$ possessing an ideal $I$; the inclusion homomorphism $I \hookrightarrow A$ is a crossed module with $A$ acting on $I$ by the multiplication in $A$, called the inclusion crossed module of algebras.

Another common instance is that of an $A$-bimodule $M$ with trivial multiplication; then the zero homomorphism $0: M \rightarrow A, m \mapsto 0$, is a crossed module.

Any epimorphism of algebras $R \rightarrow A$ with the kernel in the two-sided annihilator of $R$ is a crossed module, with $a \in A$ acting on $r \in R$ by $a r=\tilde{r} r$ and $r a=r \tilde{r}$ where $\tilde{r}$ is any element in the preimage of $a$.

A morphism $(\mu, v):(R, A, \rho) \rightarrow\left(R^{\prime}, A^{\prime}, \rho^{\prime}\right)$ of crossed modules is a commutative square of algebras

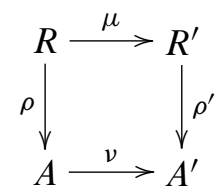


such that $\mu(a r)=v(a) \mu(r)$ and $\mu(r a)=\mu(r) v(a)$ for $a \in A, r \in R$. Let us denote the category of crossed modules of algebras by $X$ Alg. Note that there is an equivalence between the category $\mathcal{X} \mathrm{Alg}$ and the category of DG-algebras concentrated in degrees 0 and 1.

Given a crossed module $(R, A, \rho)$ of algebras, consider the semidirect product algebra, $R \rtimes A$. There are algebra homomorphisms $s: R \rtimes A \rightarrow A,(r, a) \mapsto a$, and $\mathrm{t}: R \rtimes A \rightarrow A,(r, a) \mapsto \rho(r)+a$, and a binary operation $\left(r^{\prime}, a^{\prime}\right) \circ(r, a)=\left(r+r^{\prime}, a\right)$ for all pairs $(r, a),\left(r^{\prime}, a^{\prime}\right) \in R \rtimes A$ such that $\rho(r)+a=a^{\prime}$. This composition $\circ$ with the source map $\mathfrak{s}$ and target map t constitutes an internal category in the category Alg and the nerve of its category structure forms the simplicial algebra $\mathfrak{N}_{*}(R, A, \rho)$ where $\mathfrak{N}_{n}(R, A, \rho)=R \rtimes(\ldots(R \rtimes A) \ldots)$ with $n$ semidirect factors of $R$, and face and degeneracy homomorphisms are defined by

$$
\begin{aligned}
d_{0}\left(r_{1}, \ldots, r_{n}, a\right) & =\left(r_{2}, \ldots, r_{n}, a\right), \\
d_{i}\left(r_{1}, \ldots, r_{n}, a\right) & =\left(r_{1}, \ldots, r_{i}+r_{i+1}, \ldots, r_{n}, a\right), \quad 0<i<n, \\
d_{n}\left(r_{1}, \ldots, r_{n}, a\right) & =\left(r_{1}, \ldots, r_{n-1}, \rho\left(r_{n}\right)+a\right), \\
s_{i}\left(r_{1}, \ldots, r_{n}, a\right) & =\left(r_{1}, \ldots, r_{i}, 0, r_{i+1}, \ldots, r_{n}, a\right), \quad 0 \leq i \leq n .
\end{aligned}
$$

The simplicial algebra $\mathfrak{N}_{*}(R, A, \rho)$ is called the nerve of the crossed module $(R, A, \rho)$.

2.2. Homologies of simplicial algebras. Let us recall that for a given simplicial algebra $A_{*}$ its Moore normalisation is a complex of algebras $\mathcal{N} A_{*}$ where

$$
\mathcal{N}_{n} A_{*}=\bigcap_{i=0}^{n-1} \operatorname{Ker} d_{i}^{n} \quad \text { and } \quad \partial_{n}=\left.d_{n}^{n}\right|_{\mathcal{N}_{n} A_{*}} .
$$

Note that the Moore complex of the nerve of a crossed module of algebras $(R, A, \rho)$ is trivial in dimensions $\geq 2$. In fact it is just the original crossed module up to isomorphism with $R$ in dimension 1 and $A$ in dimension 0 .

The $n$-th homotopy of the simplicial algebra is defined as

$$
\pi_{n}\left(A_{*}\right)=\operatorname{Ker} \partial_{n} / \operatorname{Im} \partial_{n+1} .
$$

Moreover, if an augmented simplicial algebra $\left(A_{*}, d_{0}^{0}, A\right)$ is given, we calculate the extended homotopy groups as $\pi_{0}\left(A_{*}, d_{0}^{0}, A\right)=\operatorname{Ker} d_{0}^{0} / \operatorname{Im} \partial_{1}$ and $\pi_{-1}\left(A_{*}, d_{0}^{0}, A\right)=$ $A / \operatorname{Im} d_{0}^{0}$. We say that the augmented simplicial algebra $\left(A_{*}, d_{0}^{0}, A\right)$ is aspherical if $\pi_{n}\left(A_{*}, d_{0}^{0}, A\right)=0$ for all $n \geq-1$.

Now we recall some required constructions of the standard complexes of algebras (e.g., see [15]) and give their extensions to simplicial algebras.

Given an algebra $A$, the standard bar complex $C^{\text {bar }}(A)$ and the Hochschild complex $C(A)$ have the form $C_{n}^{\text {bar }}(A)=C_{n}(A):=A^{\otimes(n+1)}$ where the boundary operator of the bar complex is given by

$$
b^{\prime}\left(a_{0} \otimes \cdots \otimes a_{n}\right)=\sum_{i=0}^{n-1}(-1)^{i}\left(a_{0} \otimes \cdots \otimes a_{i} a_{i+1} \otimes \cdots \otimes a_{n}\right),
$$


while the Hochschild boundary is given by

$$
b\left(a_{0} \otimes \cdots \otimes a_{n}\right)=b^{\prime}\left(a_{0} \otimes \cdots \otimes a_{n}\right)+(-1)^{n}\left(a_{n} a_{0} \otimes a_{1} \otimes \cdots \otimes a_{n-1}\right) .
$$

Consider the cyclic, first quadrant bicomplex

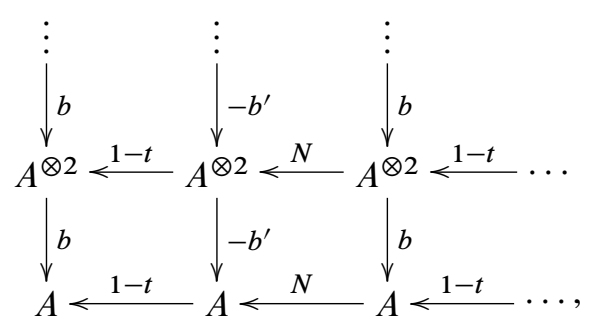

where $t: A^{\otimes(n+1)} \rightarrow A^{\otimes(n+1)}, n \geq 0$, is the cyclic operator given by $t\left(a_{0} \otimes \cdots \otimes\right.$ $\left.a_{n}\right)=(-1)^{n}\left(a_{n} \otimes a_{0} \otimes \cdots \otimes a_{n-1}\right)$ and $N: A^{\otimes(n+1)} \rightarrow A^{\otimes(n+1)}$ is the operator defined by $N=1+t+t^{2}+\cdots+t^{n}$. We denote by $C C(A)$ and $C C^{\{2\}}(A)$ the total complexes of the bicomplex (1) and the bicomplex obtained through deleting all columns whose indices are $\geq 2$ in (1), respectively.

Suppose that $A$ is an algebra and we are given a functorial chain complex $\Phi(A)$, as in the case of $C(A), C^{\mathrm{bar}}(A), C C^{\{2\}}(A)$ and $C C(A)$ complexes, and set $H_{n}^{\Phi}(A)=$ $H_{n}(\Phi(A)), n \geq 0$. This homology extends to simplicial algebras in the standard way, by applying the functor $\Phi$ dimension-wise to a given simplicial algebra $A_{*}$ and then taking the homology of the total complex of resulting bicomplex $\Phi\left(A_{*}\right)$. This homology is denoted by $H_{n}^{\Phi}\left(A_{*}\right), n \geq 0$.

Now let $(R, A, \rho)$ be a crossed module of algebras and $\Phi(R, A, \rho)$ denote the total complex $\operatorname{Tot}\left(\Phi\left(\Re_{*}(R, A, \rho)\right)\right)$. Then the Hochschild, bar, naive Hochschild and cyclic homologies of $(R, A, \rho)$ are defined by

$$
\begin{aligned}
\mathrm{HH}_{n}(R, A, \rho) & =H_{n}\left(C C^{\{2\}}(R, A, \rho)\right), & H_{n}^{\mathrm{bar}}(R, A, \rho) & =H_{n}\left(C^{\mathrm{bar}}(R, A, \rho)\right), \\
\mathrm{HH}_{n}^{\text {naive }}(R, A, \rho) & =H_{n}(C(R, A, \rho)), & & \mathrm{HC}_{n}(R, A, \rho)=H_{n}(C C(R, A, \rho))
\end{aligned}
$$

for $n \geq 0$.

Clearly, for any simplicial algebra $A_{*}$ there is a short exact sequence of complexes

$$
0 \rightarrow \operatorname{Tot}\left(C C^{\{2\}}\left(A_{*}\right)\right) \rightarrow \operatorname{Tot}\left(C C\left(A_{*}\right)\right) \rightarrow \operatorname{Tot}\left(C C\left(A_{*}\right)\right)_{-2} \rightarrow 0,
$$

where $\operatorname{Tot}\left(C C_{n}\left(A_{*}\right)\right)_{-2}=\operatorname{Tot}\left(C C_{n-2}\left(A_{*}\right)\right)$. This gives rise to an analogue of Connes' periodicity exact sequence for simplicial algebras:

$$
\cdots \rightarrow \mathrm{HH}_{n}\left(A_{*}\right) \stackrel{I}{\rightarrow} \mathrm{HC}_{n}\left(A_{*}\right) \stackrel{S}{\rightarrow} \mathrm{HC}_{n-2}\left(A_{*}\right) \stackrel{B}{\rightarrow} \mathrm{HH}_{n-1}\left(A_{*}\right) \rightarrow \cdots
$$

The following facts about homology of aspherical augmented simplicial algebras will be useful in the sequel. 
Lemma 2.3. Let $\left(A_{*}, d_{0}^{0}, A\right)$ be an aspherical augmented simplicial algebra and $\Phi:$ Alg $\rightarrow \mathfrak{C}_{\geq 0}$ be a covariant functor. Let $\widetilde{\Phi}_{n}$ : Vect $\rightarrow$ Vect, $n \geq 0$, be a functor such that $\Phi_{n}=\widetilde{\Phi}_{n} \circ W$ where $W:$ Alg $\rightarrow$ Vect is the usual forgetful functor. Then

(i) the augmented simplicial vector space $\left(\Phi_{n}\left(A_{*}\right), \Phi_{n}\left(d_{0}^{0}\right), \Phi_{n}(A)\right)$ is acyclic for $n \geq 0$;

(ii) there is a natural isomorphism

$$
H_{n}^{\Phi}(A) \cong H_{n}^{\Phi}\left(A_{*}\right), \quad n \geq 0 .
$$

Proof. (i) Straightforward from the fact that an acyclic augmented simplicial vector space $\left(W\left(A_{*}\right), W\left(d_{0}^{0}\right), W(A)\right)$ has a linear left (right) contraction.

(ii) Let us consider the bicomplex $\Phi\left(A_{*}\right)$. Using (i), for any fixed $q$ the (horizontal) homology of the bicomplex $\Phi\left(A_{*}\right)$ is $H_{p}\left(\Phi_{q}\left(A_{*}\right)\right)=0, p>0$, and $H_{0}\left(\Phi_{q}\left(A_{*}\right)\right)=$ $\Phi_{q}(A)$. Now the bicomplex spectral sequence argument completes the proof.

Corollary 2.4. Let $\left(A_{*}, d_{0}^{0}, A\right)$ be an aspherical augmented simplicial algebra. Then, for $n \geq 0$, there are natural isomorphisms

$$
\begin{aligned}
\mathrm{HH}_{n}\left(A_{*}\right) & \cong \mathrm{HH}_{n}(A), \quad \operatorname{HH}_{n}^{\mathrm{bar}}\left(A_{*}\right) \cong \mathrm{HH}_{n}^{\mathrm{bar}}(A), \\
\operatorname{HH}_{n}^{\text {naive }}\left(A_{*}\right) & \cong \mathrm{HH}_{n}^{\text {naive }}(A), \quad \operatorname{HC}_{n}\left(A_{*}\right) \cong \mathrm{HC}_{n}(A) .
\end{aligned}
$$

Proof. It is clear that the values of the functors $C C_{n}^{\{2\}}, C_{n}^{\text {bar }}, C_{n}$ and $C C_{n}, n \geq 0$, on the algebra $A$ depend only on the vector space underlying $A$. Due to Lemma 2.3 (ii) the proof is completed.

Note that if we are given an inclusion crossed module of algebras $I \hookrightarrow A$, then $\left(\Re_{*}(I \hookrightarrow A), d_{0}^{0}, A / I\right)$ is an aspherical augmented simplicial algebra and by Corollary 2.4, any of its homology theories mentioned above coincides with the respective homology of $A / I$.

\section{Hochschild and cyclic homologies of crossed modules}

In this section we study some properties of the Hochschild and cyclic homologies of crossed modules of algebras.

3.1. Five-term exact sequences. Now we establish the five-term exact sequences relating the Hochschild and cyclic homologies of crossed modules of algebras and their cokernel algebras. 
Theorem 3.2. Let $(R, A, \rho)$ be a crossed module of algebras. There are exact sequences of vector spaces

$$
\begin{aligned}
\mathrm{HH}_{2}(R, A, \rho) \rightarrow \mathrm{HH}_{2}(\operatorname{Coker} \rho) & \rightarrow \operatorname{Ker} \rho /[A, \operatorname{Ker} \rho] \\
& \rightarrow \mathrm{HH}_{1}(R, A, \rho) \rightarrow \mathrm{HH}_{1}(\text { Coker } \rho) \rightarrow 0, \\
\mathrm{HC}_{2}(R, A, \rho) \rightarrow \mathrm{HC}_{2}(\operatorname{Coker} \rho) & \rightarrow \operatorname{Ker} \rho /[A, \operatorname{Ker} \rho] \\
& \rightarrow \mathrm{HC}_{1}(R, A, \rho) \rightarrow \mathrm{HC}_{1}(\text { Coker } \rho) \rightarrow 0,
\end{aligned}
$$

and the equality $\mathrm{HH}_{0}(R, A, \rho)=\operatorname{HC}_{0}(R, A, \rho)=\operatorname{Coker} \rho /[$ Coker $\rho$, Coker $\rho]$ holds.

Proof. We will only prove the exactness of the first sequence. The proof for the second is essentially the same and left to the reader.

Consider the bicomplex $C C^{\{2\}}\left(\Re_{*}(R, A, \rho)\right)$. Then there is a first quadrant spectral sequence

$$
E_{p q}^{1}=H_{q}\left(C C_{p}^{\{2\}}\left(\Re_{*}(R, A, \rho)\right)\right) \Longrightarrow \mathrm{HH}_{p+q}(R, A, \rho) .
$$

It is easy to check that $E_{p 0}^{1}=C C_{p}^{\{2\}}(\operatorname{Coker} \rho), p \geq 0$ and $E_{01}^{1}=\pi_{1}\left(\Re_{*}(R, A, \rho)\right)=$ Ker $\rho$. Using the Eilenberg-Zilber theorem and the Künneth formula we have

$$
\begin{aligned}
E_{11}^{1}= & \left(\pi_{1}\left(\Re_{*}(R, A, \rho)\right) \otimes \pi_{0}\left(\Re_{*}(R, A, \rho)\right)\right) \\
& \oplus\left(\pi_{0}\left(\Re_{*}(R, A, \rho)\right) \otimes \pi_{1}\left(\mathfrak{N}_{*}(R, A, \rho)\right)\right) \oplus \pi_{1}\left(\mathfrak{N}_{*}(R, A, \rho)\right) \\
= & (\operatorname{Ker} \rho \otimes \operatorname{Coker} \rho) \oplus(\operatorname{Coker} \rho \otimes \operatorname{Ker} \rho) \oplus \operatorname{Ker} \rho .
\end{aligned}
$$

Continuing calculations we deduce that $E_{00}^{\infty}=E_{00}^{2}=\mathrm{HH}_{0}(\operatorname{Coker} \rho)$ and $E_{10}^{\infty}=$ $E_{10}^{2}=\mathrm{HH}_{1}(\operatorname{Coker} \rho)$. Moreover,

$$
E_{01}^{2}=\operatorname{Coker}\left\{E_{11}^{1} \rightarrow E_{01}^{1}\right\}=\operatorname{Ker} \rho /[\operatorname{Ker} \rho, \operatorname{Coker} \rho]=\operatorname{Ker} \rho /[A, \operatorname{Ker} \rho] .
$$

Therefore, we have a differential $d^{2}: \mathrm{HH}_{2}(\operatorname{Coker} \rho) \rightarrow \operatorname{Ker} \rho /[A, \operatorname{Ker} \rho]$ of the spectral sequence, which determines the base term $E_{20}^{\infty}$ and the fiber term $E_{01}^{\infty}$ from the following exact sequence:

$$
0 \rightarrow E_{20}^{\infty} \rightarrow \mathrm{HH}_{2}(\text { Coker } \rho) \rightarrow \operatorname{Ker} \rho /[A, \operatorname{Ker} \rho] \rightarrow E_{01}^{\infty} \rightarrow 0 .
$$

Clearly, we have the short exact sequence

$$
0 \rightarrow E_{01}^{\infty} \rightarrow \mathrm{HH}_{1}(R, A, \rho) \rightarrow E_{10}^{\infty} \rightarrow 0
$$

and the epimorphism

$$
\mathrm{HH}_{2}(R, A, \rho) \rightarrow E_{20}^{\infty} .
$$

Now (3), (4) and (5) imply the required result.

Theorem 3.2 shows that the Hochschild (resp. cyclic) homology of a crossed module $(R, A, \rho)$ differs, in general, from the Hochschild (resp. cyclic) homology of the cokernel algebra Coker $\rho$. 
3.3. Excision property. In this subsection we discuss some aspects of the excision property for Hochschild (resp. cyclic) homology of crossed modules. Namely, we extend Wodzicki's excision theorem [20] for inclusion crossed modules in the category of crossed modules of algebras.

The excision problem for Hochschild (resp. cyclic) homology in the category of crossed module of algebras is formulated as follows: let

$$
0 \rightarrow(R, A, \rho) \stackrel{(\mu, \nu)}{\longrightarrow}(S, B, \sigma) \stackrel{(\eta, \theta)}{\longrightarrow}(T, C, \tau) \rightarrow 0
$$

be a linearly split extension of crossed modules of algebras, i.e., a sequence of crossed modules such that $0 \rightarrow R \stackrel{\mu}{\rightarrow} S \stackrel{\eta}{\rightarrow} T \rightarrow 0$ and $0 \rightarrow A \stackrel{v}{\rightarrow} B \stackrel{\theta}{\rightarrow} C \rightarrow 0$ are exact sequences of algebras, with an existing pair of linear maps $\gamma: T \rightarrow S$ and $\delta: C \rightarrow B$ such that $\eta \gamma=1_{T}, \theta \delta=1_{C}$ and $\sigma \gamma=\delta \tau$. The crossed module $(R, A, \rho)$ is excisive (or satisfies excision) for Hochschild (resp. cyclic) homology if the induced natural map

$$
C C^{\{2\}}(R, A, \rho) \rightarrow \operatorname{Ker}\left\{C C^{\{2\}}(S, B, \sigma) \rightarrow C C^{\{2\}}(T, C, \tau)\right\}
$$

(resp. $C C(R, A, \rho) \rightarrow \operatorname{Ker}\{C C(S, B, \sigma) \rightarrow C C(T, C, \tau)\})$ is a quasi-isomorphism for any linearly split extension (6) of the crossed module $(R, A, \rho)$.

Note that according to Connes' periodicity exact sequence (2), the excision properties for Hochschild and cyclic homologies in the category of crossed modules of algebras are equivalent.

The aim of this subsection is to prove the following.

Theorem 3.4. Let $(I, A$, inc) be an inclusion crossed module of algebras. The following conditions are equivalent:

(i) $(I, A$, inc) is excisive for Hochschild homology,

(ii) $H_{n}^{\text {bar }}(I, A$,inc $)=0$ for all $n \geq 0$.

Proof. (i) $\Longrightarrow$ (ii) Consider the algebra $\boldsymbol{k}^{\prime}$ with the underlying vector space $\boldsymbol{k}$ endowed with the trivial multiplication and the inclusion crossed module of algebras $\left(I, A \times \boldsymbol{k}^{\prime}\right.$, inc $\left.\times 0\right)$. Then there is a linearly split extension of crossed modules of algebras

$$
0 \rightarrow(I, A \text {, inc }) \rightarrow\left(I, A \times \boldsymbol{k}^{\prime}, \text { inc } \times 0\right) \rightarrow\left(0, \boldsymbol{k}^{\prime}, 0\right) \rightarrow 0 .
$$

Hence the induced map

$$
C C^{\{2\}}(I, A, \text { inc }) \rightarrow \operatorname{Ker}\left\{C C^{\{2\}}\left(I, A \times \boldsymbol{k}^{\prime}, \text { inc } \times 0\right) \rightarrow C C^{\{2\}}\left(0, \boldsymbol{k}^{\prime}, 0\right)\right\}
$$

is a quasi-isomorphism. It is easy to check that the cokernel of this map contains $C^{\text {bar }}(I, A$, inc $)$ as a direct summand. This implies the result. 
(ii) $\Longrightarrow$ (i) Consider any linearly split extension (6) of crossed modules of algebras. Hence we have the short exact sequence

$$
\begin{aligned}
& 0 \rightarrow \pi_{0}\left(\Re_{*}(I, A, \text { inc })\right) \stackrel{\pi_{0}\left(\Re_{*}(\mu, \nu)\right)}{\longrightarrow} \pi_{0}\left(\Re_{*}(S, B, \sigma)\right) \\
& \stackrel{\pi_{0}\left(\Re_{*}(\eta, \theta)\right)}{\longrightarrow} \pi_{0}\left(\Re_{*}(T, C, \tau)\right) \rightarrow 0
\end{aligned}
$$

and the isomorphism

$$
\pi_{1}\left(\Re_{*}(S, B, \sigma)\right) \stackrel{\pi_{1}\left(\Re_{*}(\eta, \theta)\right)}{\cong} \pi_{1}\left(\Re_{*}(T, C, \tau)\right) .
$$

It is also easy to see that we have the following commutative diagram with exact rows of complexes:

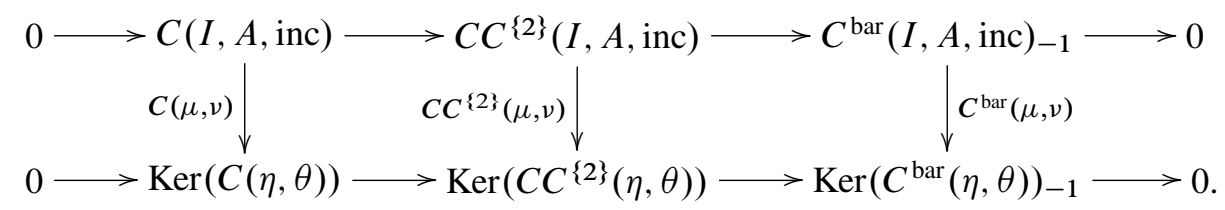

Here we have $C_{n}^{\text {bar }}(I, A \text {, inc })_{-1}=C_{n-1}^{\text {bar }}(I, A$, inc $)$ and $\operatorname{Ker}_{n}\left\{C^{\text {bar }}(\eta, \theta)\right\}_{-1}=$ $\operatorname{Ker}_{n-1}\left\{C^{\text {bar }}(\eta, \theta)\right\}$. Clearly, the induced commutative diagram of long exact homology sequences and the five lemma imply that if $C(\mu, v)$ and $C^{\text {bar }}(\mu, v)$ are quasiisomorphisms, then so is $C C^{\{2\}}(\mu, v)$.

We shall only prove that $C(\mu, v)$ is a quasi-isomorphism. The fact that $C^{\mathrm{bar}}(\mu, v)$ is a quasi-isomorphism can be proved essentially in the same way and will be omitted.

We need the following.

Lemma 3.5. Let $(S, B, \sigma)$ be any crossed module of algebras, then there is an isomorphism

$$
\begin{aligned}
& H_{q}\left(C_{p}\left(\Re_{*}(S, B, \sigma)\right)\right) \\
& \cong\left\{\begin{array}{l}
\bigoplus_{i_{0}+\cdots+i_{p}=q}\left(\pi_{i_{0}}\left(\Re_{*}(S, B, \sigma)\right) \otimes \cdots \otimes \pi_{i_{p}}\left(\Re_{*}(S, B, \sigma)\right)\right), \\
0, \quad 0 \leq p<q-1,
\end{array} \quad \begin{array}{l}
p \geq 0 \leq q \leq p+1,
\end{array}\right.
\end{aligned}
$$

with $i_{0}, \ldots, i_{p}=0$ or 1 . Moreover, through this isomorphism the Hochschild differential behaves as follows:

$x_{0} \otimes x_{1} \otimes \cdots \otimes x_{p}$

$$
\mapsto \sum_{j=0}^{p-1}(-1)^{j}\left(x_{0} \otimes \cdots \otimes x_{j} x_{j+1} \otimes \cdots \otimes x_{p}\right)+(-1)^{p}\left(x_{p} x_{0} \otimes \cdots \otimes x_{p-1}\right) .
$$

Here $x_{j} \in \pi_{i_{j}}\left(\Re_{*}(S, B, \sigma)\right), 0 \leq j \leq p$, and the multiplication $x_{j} x_{j^{\prime}}$ is understood as the multiplication in $\pi_{0}\left(\Re_{*}(S, B, \sigma)\right)$ or the $\pi_{0}\left(\Re_{*}(S, B, \sigma)\right)$-bimodule structure on $\pi_{1}\left(\mathfrak{N}_{*}(S, B, \sigma)\right)$. 
Proof. The proof, requiring to use again the Eilenberg-Zilber theorem and the Künneth formula, is routine and will be omitted.

Returning to the main proof, consider the bicomplexes $C\left(\Re_{*}(I, A\right.$, inc $\left.)\right)$ and $\mathrm{M} \equiv$ $\operatorname{Ker}\left(C\left(\Re_{*}(S, B, \sigma)\right) \stackrel{C\left(\Re_{*}(\eta, \theta)\right)}{\longrightarrow} C\left(\Re_{*}(T, C, \tau)\right)\right)$. Then there are first quadrant spectral sequences

$$
E_{p q}^{1}=H_{q}\left(C_{p}\left(\Re_{*}(I, A, \text { inc })\right)\right) \Longrightarrow \operatorname{HH}_{p+q}^{\text {naive }}(I, A, \text { inc })
$$

and

$$
\begin{aligned}
\bar{E}_{p q}^{1}=H_{q}\left(\operatorname { K e r } \left(C_{p}\left(\Re_{*}(S, B, \sigma)\right)\right.\right. \\
\left.\left.\quad \stackrel{C_{p}\left(\Re_{*}(\eta, \theta)\right)}{\longrightarrow} C_{p}\left(\Re_{*}(T, C, \tau)\right)\right)\right) \Longrightarrow H_{p+q}(\operatorname{Tot}(\mathbb{M})) .
\end{aligned}
$$

Moreover, we have the linearly split exact sequence of simplicial vector spaces

$$
0 \rightarrow \mathbb{M}_{* p} \rightarrow C_{p}\left(\Re_{*}(S, B, \sigma)\right) \rightarrow C_{p}\left(\Re_{*}(T, C, \tau)\right) \rightarrow 0,
$$

implying the short exact homology sequence

$$
0 \rightarrow \bar{E}_{p q}^{1} \rightarrow H_{q}\left(C_{p}\left(\Re_{*}(S, B, \sigma)\right)\right) \rightarrow H_{q}\left(C_{p}\left(\Re_{*}(T, C, \tau)\right)\right) \rightarrow 0 .
$$

Hence, from Lemma 3.5 we have an isomorphism

$$
\bar{E}_{p q}^{1} \cong \begin{cases}\bigoplus_{i_{0}+\cdots+i_{p}=q} \operatorname{Ker}\left\{\pi_{i_{0}}\left(\Re_{*}(\eta, \theta)\right) \otimes \cdots \otimes \pi_{i_{p}}\left(\Re_{*}(\eta, \theta)\right)\right\} \\ 0 \quad \text { for } 0 \leq p<q-1, & \text { for } p \geq 0,0 \leq q \leq p+1,\end{cases}
$$

with $i_{0}, \ldots, i_{p}=0$ or 1 .

There is a natural morphism of bicomplexes $C\left(\Re_{*}(\mu, \nu)\right): C\left(\mathfrak{N}_{*}(I, A\right.$, inc $\left.)\right) \rightarrow$ $M$, inducing the morphism of spectral sequences $f^{1}: E^{1} \rightarrow \bar{E}^{1}$. To finish the proof it suffices to show that $f_{p q}^{2}: E_{p q}^{2} \rightarrow \bar{E}_{p q}^{2}$ is an isomorphism for any $p, q \geq 0$.

We prove the remaining part of the assertion in two cases.

Case 1. The morphism $f_{p q}^{2}: E_{p q}^{2} \rightarrow \bar{E}_{p q}^{2}$ is an isomorphism for any $p \geq 0$ and $q=0$.

Indeed, by Lemma 3.5 we have $E_{p 0}^{2}=\operatorname{HH}_{p}^{\text {naive }}\left(\pi_{0}\left(\Re_{*}(I, A\right.\right.$, inc $\left.\left.)\right)\right)$, while by (9)

$$
\bar{E}_{p 0}^{2} \cong H_{p}\left(\operatorname{Ker}\left\{C\left(\pi_{0}\left(\Re_{*}(S, B, \sigma)\right)\right) \stackrel{C\left(\pi_{0}\left(\Re_{*}(\eta, \theta)\right)\right)}{\longrightarrow} C\left(\pi_{0}\left(\Re_{*}(T, C, \tau)\right)\right)\right\}\right) .
$$

By the assumption on the crossed module $(I, A$, inc) and Corollary 2.4 the algebra $\pi_{0}\left(\Re_{*}(I, A\right.$, inc $\left.)\right)$ is $H$-unital, then the result follows from Wodzicki's theorem [20] applied to the short exact sequence of algebras (7).

Case 2. The morphism $f_{p q}^{2}: E_{p q}^{2} \rightarrow \bar{E}_{p q}^{2}$ is an isomorphism for any $p \geq 0$ and $q>0$. 
Using Lemma 3.5 again, we have $E_{p q}^{1}=0$ and consequently $E_{p q}^{2}=0$ for any $p \geq 0$ and $q>0$. Thus, we are left to show that $\bar{E}_{p q}^{2}=0, p \geq 0$ and $q>0$. Indeed, by (7) and (8) we have the short exact sequence of algebras

$$
\begin{aligned}
0 \rightarrow \pi_{0}\left(\mathfrak{N}_{*}(I, A, \text { inc })\right) & \rightarrow \pi_{1}\left(\mathfrak{N}_{*}(S, B, \sigma)\right) \rtimes \pi_{0}\left(\mathfrak{N}_{*}(S, B, \sigma)\right) \\
& \rightarrow \pi_{1}\left(\mathfrak{N}_{*}(T, C, \tau)\right) \rtimes \pi_{0}\left(\mathfrak{N}_{*}(T, C, \tau)\right) \rightarrow 0 .
\end{aligned}
$$

Hence, the fact that $\pi_{0}\left(\Re_{*}(I, A\right.$, inc $\left.)\right)$ is $H$-unital, by Wodzicki's theorem [20], implies that we have the quasi-isomorphism

$$
\begin{aligned}
C\left(\pi_{0}\left(\Re_{*}(I, A, \text { inc })\right)\right) & \rightarrow \operatorname{Ker}\left\{C\left(\pi_{1}\left(\Re_{*}(S, B, \sigma)\right) \rtimes \pi_{0}\left(\Re_{*}(S, B, \sigma)\right)\right)\right. \\
& \left.\rightarrow C\left(\pi_{1}\left(\Re_{*}(T, C, \tau)\right) \rtimes \pi_{0}\left(\Re_{*}(T, C, \tau)\right)\right)\right\} .
\end{aligned}
$$

Moreover, we have the isomorphisms of vector spaces

$$
\begin{aligned}
C_{p}\left(\pi_{1}\left(\Re_{*}(S, B, \sigma)\right) \rtimes \pi_{0}\left(\Re_{*}(S, B, \sigma)\right)\right) \\
\quad \cong\left(\pi_{0}\left(\Re_{*}(S, B, \sigma)\right)\right)^{\otimes p+1} \\
\quad \underset{i_{0}+\cdots+i_{p}=1}{\bigoplus_{i_{0}}^{p+1}}\left(\pi_{i_{0}}\left(\Re_{*}(S, B, \sigma)\right) \otimes \cdots \otimes \pi_{i_{p}}\left(\Re_{*}(S, B, \sigma)\right)\right)
\end{aligned}
$$

and

$$
\begin{aligned}
C_{p}\left(\pi_{1}\left(\Re_{*}(T, C, \tau)\right) \rtimes \pi_{0}\left(\Re_{*}(T, C, \tau)\right)\right) \\
\quad \cong\left(\pi_{0}\left(\Re_{*}(T, C, \tau)\right)\right)^{\otimes p+1} \\
\quad \oplus \bigoplus_{i_{0}+\cdots+i_{p}=1}^{p+1}\left(\pi_{i_{0}}\left(\Re_{*}(T, C, \tau)\right) \otimes \cdots \otimes \pi_{i_{p}}\left(\Re_{*}(T, C, \tau)\right)\right),
\end{aligned}
$$

with $i_{0}, \ldots, i_{p}=0$ or 1 .

Let us define $\mathfrak{D}_{p}^{q}$ and $\overline{\mathfrak{D}}_{p}^{q}$ for any $p \geq 0$ and $q>0$ by the formulas

$$
\mathfrak{D}_{p}^{q}= \begin{cases}\bigoplus_{i_{0}+\cdots+i_{p}=q}\left(\pi_{i_{0}}\left(\mathfrak{N}_{*}(S, B, \sigma)\right) \otimes \cdots \otimes \pi_{i_{p}}\left(\mathfrak{N}_{*}(S, B, \sigma)\right)\right) & \text { for } q \leq p+1, \\ 0 \text { for } p+1<q, & \end{cases}
$$

and

$$
\overline{\mathfrak{D}}_{p}^{q}= \begin{cases}\bigoplus_{i_{0}+\cdots+i_{p}=q}\left(\pi_{i_{0}}\left(\mathfrak{N}_{*}(T, C, \tau)\right) \otimes \cdots \otimes \pi_{i_{p}}\left(\mathfrak{N}_{*}(T, C, \tau)\right)\right) \\ 0 \text { for } p+1<q, & \text { for } q \leq p+1,\end{cases}
$$

with $i_{0}, \ldots, i_{p}=0$ or 1 .

It is not difficult to show that $\mathfrak{D}^{q}$ is a subcomplex of $C\left(\pi_{1}\left(\Re_{*}(S, B, \sigma)\right) \rtimes\right.$ $\left.\pi_{0}\left(\Re_{*}(S, B, \sigma)\right)\right)$ through the isomorphism (11) and along the Hochschild differential, which is left to the reader. (Hint: The induced multiplication in $\pi_{1}\left(\mathfrak{N}_{*}(S, B, \sigma)\right)$ 
vanishes.) Similarly, $\overline{\mathfrak{D}}^{q}$ is a subcomplex of $C\left(\pi_{1}\left(\mathfrak{N}_{*}(T, C, \tau)\right) \rtimes \pi_{0}\left(\mathfrak{N}_{*}(T, C, \tau)\right)\right)$. Moreover, by (11) and (12), there are decompositions in direct summands of complexes

$$
C\left(\pi_{1}\left(\Re_{*}(S, B, \sigma)\right) \rtimes \pi_{0}\left(\Re_{*}(S, B, \sigma)\right)\right) \cong C\left(\pi_{0}\left(\Re_{*}(S, B, \sigma)\right)\right) \oplus \underset{q \geq 1}{\bigoplus} \mathfrak{D}^{q}
$$

and

$$
C\left(\pi_{1}\left(\Re_{*}(T, C, \tau)\right) \rtimes \pi_{0}\left(\Re_{*}(T, C, \tau)\right)\right) \cong C\left(\pi_{0}\left(\Re_{*}(T, C, \tau)\right)\right) \oplus \bigoplus_{q \geq 1} \overline{\mathfrak{D}}^{q} .
$$

Hence

$$
\begin{aligned}
& \operatorname{Ker}\left\{C\left(\pi_{1}\left(\Re_{*}(S, B, \sigma)\right) \rtimes \pi_{0}\left(\Re_{*}(S, B, \sigma)\right)\right)\right. \\
& \left.\quad \rightarrow C\left(\pi_{1}\left(\Re_{*}(T, C, \tau)\right) \rtimes \pi_{0}\left(\Re_{*}(T, C, \tau)\right)\right)\right\} \cong \operatorname{Ker}\left\{C\left(\pi_{0}\left(\Re_{*}(S, B, \sigma)\right)\right)\right. \\
& \left.\quad \stackrel{\pi_{0}\left(\Re_{*}(\eta, \theta)\right)}{\longrightarrow} C\left(\pi_{0}\left(\Re_{*}(T, C, \tau)\right)\right)\right\} \oplus \underset{q \geq 1}{\bigoplus \operatorname{Ker}}\left\{\mathfrak{D}^{q} \rightarrow \overline{\mathfrak{D}}^{q}\right\} .
\end{aligned}
$$

Now the quasi-isomorphism (10) implies that the complex $\operatorname{Ker}\left(\mathfrak{D}^{q} \rightarrow \overline{\mathfrak{D}}^{q}\right)$ is acyclic for any $q>0$, which according to (9) means that $\bar{E}_{p q}^{2}=0$ for any $p \geq 0$ and $q>0$.

\section{Cotriple cyclic homology of crossed modules}

In this section we demonstrate a construction of a (new) cotriple cyclic homology theory of crossed modules of algebras, using the theory of non-abelian derived functors.

During this section we assume that $\mathbb{k}$ is a field of characteristic zero.

4.1. Adjunction. We begin by constructing a pair of functors $\operatorname{Alg} \underset{U}{\stackrel{F}{\underset{ }{\rightleftarrows}}} \mathcal{X} \mathrm{Alg}$ as follows: For any crossed module $(R, A, \rho)$, let $U(R, A, \rho)$ denote the direct product $R \times A$, and for any algebra $A$, let $F(A)$ denote the inclusion crossed module of algebras ( $\bar{A}, A * A$, inc), where $A * A$ is the coproduct of the algebra $A$ with itself, with inclusions $u_{1}, u_{2}: A \rightarrow A * A$, and $\bar{A}$ is the kernel of the retraction $p_{2}: A * A \rightarrow A$ determined by the conditions $p_{2} u_{2}=1_{A}, p_{2} u_{1}=0$.

Proposition 4.2. The functor $F$ is left adjoint to the functor $U$.

Proof. We state that, given an algebra $A$, the homomorphism

$$
\left(u_{1}, u_{2}\right): A \rightarrow \bar{A} \times(A * A)=U F(A)
$$

is a universal arrow from $A$ to the functor $U$. Indeed, let $(S, B, \sigma)$ be a crossed module and $f_{S}: A \rightarrow S, f_{B}: A \rightarrow B$ defining homomorphisms of any homomorphism 
$\left(f_{S}, f_{B}\right): A \rightarrow S \times B=U(S, B, \sigma)$. Then there is a commutative diagram with split short exact sequences of algebras,

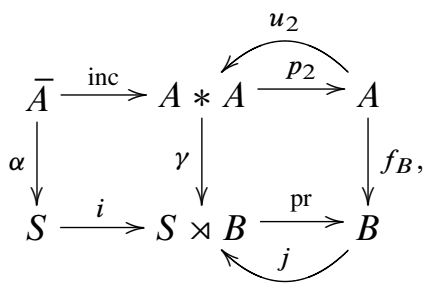

where $\gamma$ is defined by $\gamma u_{1}=i f_{S}$ and $\gamma u_{2}=j f_{B}$, and $\alpha$ is the restriction of $\gamma$. Let $\beta: A * A \rightarrow B$ be the unique homomorphism satisfying $\beta u_{1}=\sigma f_{S}$ and $\beta u_{2}=f_{B}$. Easy calculations show that $(\alpha, \beta):(\bar{A}, A * A$, inc $) \rightarrow(S, B, \sigma)$ is a morphism of crossed modules of algebras, which is clearly the unique one such that $(\alpha \times \beta)\left(u_{1}, u_{2}\right)=\left(f_{S}, f_{B}\right)$.

We denote by $W:$ Alg $\rightarrow$ Vect the usual forgetful functor and by $T:$ Vec $\rightarrow \operatorname{Alg}$ its left adjoint functor, carrying any vector space $V$ to the free non-unital algebra on it. Composing these two adjunctions, Vect $\underset{W}{\stackrel{T}{\rightleftarrows}} \operatorname{Alg} \underset{U}{\stackrel{F}{\underset{ }{\rightleftarrows}}}$ XAlg, we deduce the following.

Proposition 4.3. The functor $\mathbb{F}=F \circ T:$ Vec $\rightarrow X \mathrm{Alg}, V \mapsto(\overline{T(V)}, T(V) *$ $T(V)$, inc), is left adjoint to the functor $\mathbb{U}=W \circ U: X \operatorname{Alg} \rightarrow \operatorname{Vect},(R, A, \rho) \mapsto$ $R \times A$.

4.4. Construction and elementary properties. It is known due to [10] (see also [7], [13]) that the cyclic homology of algebras is described as the non-abelian derived functors of the additive abelianisation functor $\mathrm{Ab}^{\text {add }}: \mathrm{Alg} \rightarrow \operatorname{Vect}, \mathrm{Ab}^{\text {add }}(A)=$ $A /[A, A]$. To generalise the cyclic homology theory to the category $\mathcal{X}$ Alg in terms of non-abelian derived functors we need to extend the additive abelianisation functor to this category. For this reason we look at the functor $\mathrm{Ab}^{\text {add }}$ as a factorisation through the category of Lie algebras Lie. Explicitly, there is an equality $\mathrm{Ab}^{\text {add }}=\mathrm{Ab} \circ \mathfrak{L}$ where $\mathbb{R}: \mathrm{Alg} \rightarrow$ Lie is the classical Liesation functor and $\mathrm{Ab}: \mathrm{Lie} \rightarrow$ Vect is the abelianisation functor of Lie algebras.

Let us construct the natural extension of the functors $\mathbb{L}$ and $\mathrm{Ab}$. We recall from [14] that a crossed module of Lie algebras $(M, G, \mu)$ is a Lie homomorphism $\mu: M \rightarrow G$ together with a bilinear map $G \times M \rightarrow M,(g, m) \mapsto{ }^{g} m$ satisfying

$$
{ }^{\left[g, g^{\prime}\right]} m={ }^{g}\left({ }^{g^{\prime}} m\right)-{ }^{g^{\prime}}\left({ }^{g} m\right), \quad{ }^{g}\left[m, m^{\prime}\right]=\left[{ }^{g} m, m^{\prime}\right]+\left[m,{ }^{g} m^{\prime}\right]
$$

such that the following conditions hold:

(i) $\mu\left(g_{m}\right)=[g, \mu(m)]$,

(ii) ${ }^{\mu(m)} m^{\prime}=\left[m, m^{\prime}\right]$ for all $m, m^{\prime} \in M, g, g^{\prime} \in G$. 
Let $X$ Lie denote the category of crossed modules of Lie algebras. Then there is a naturally defined functor $\mathcal{X} \mathfrak{L}: \mathcal{X} \mathrm{Alg} \rightarrow \mathcal{X}$ Lie carrying a crossed module of algebras $\rho: R \rightarrow A$ to the crossed module of Lie algebras $\mathfrak{L}(\mu): \mathfrak{L}(R) \rightarrow \mathfrak{L}(A)$ with $^{a} r=a r-r a$. Moreover, it is known that an abelian group object in the category $X$ Lie is just a linear map of vector spaces, and let $X$ Vect denote their category. Now the abelianisation of crossed modules of Lie algebras $X \mathrm{Ab}: X_{\text {Lie }} \rightarrow X$ Vect is left adjoint to the natural embedding functor $X$ Vect $\subset X$ Lie.

From the aforementioned discussion we arrive to the definition of the additive abelianisation functor of crossed modules of algebras $X_{\mathrm{Ab}^{\text {add }}}: X \mathrm{Alg} \rightarrow X$ Vect as the composition $X \mathrm{Ab}^{\text {add }}=X \mathrm{Ab} \circ \mathcal{X} \mathrm{R}$. Hence, for a crossed module $(R, A, \rho)$, we have $\mathcal{X} \mathrm{Ab}^{\text {add }}(R, A, \rho)=(R /[A, R], A /[A, A], \bar{\rho})$, where $\bar{\rho}$ is the linear map induced by $\rho$.

Now we are ready to construct the cotriple cyclic homology of crossed modules of algebras. We assume the reader is familiar with cotriples and projective classes. See, for example, [1] and [12], Chapter 2, for the background. The adjoint pair of functors Vect $\underset{\mathbb{U}}{\stackrel{\mathbb{F}}{\rightleftarrows}} X$ Alg induces a cotriple $\mathscr{F}=(\mathscr{F}, \delta, \tau)$ in $X$ Alg by the obvious way: $\mathscr{F}=\mathbb{F} \mathbb{U}: X \operatorname{Alg} \rightarrow X \operatorname{Alg}, \tau: \mathscr{F} \rightarrow 1 X_{\text {Alg }}$ is the counit and $\delta=\mathbb{F} u \mathbb{U}: \mathscr{F}^{\rightarrow} \mathscr{F}^{2}$ where $u: 1_{\text {Vect }} \rightarrow \mathbb{U F}$ is the unit of the adjunction. Let $\mathcal{P}$ denote the projective class induced by the cotriple $\mathcal{F}:(R, A, \rho) \in \mathcal{P}$ iff there exists a morphism $\vartheta:(R, A, \rho) \rightarrow$ $\mathcal{F}(R, A, \rho)$ such that $\tau_{(R, A, \rho)} \vartheta=1_{(R, A, \rho)}$.

Given any crossed module $(R, A, \rho)$, there is an augmented simplicial object $\mathcal{F}_{*}(R, A, \rho) \rightarrow(R, A, \rho)$ in the category $X$ Alg where

$$
\begin{aligned}
& \mathscr{F}_{n}(R, A, \rho)=\mathscr{F}^{n+1}(R, A, \rho)=\mathscr{F}\left(\mathscr{F}^{n}(R, A, \rho)\right), \\
& d_{i}^{n}=\mathscr{F}^{i}\left(\tau_{\mathcal{F}^{n-i}}\right), \quad s_{i}^{n}=\mathscr{F}^{i}\left(\delta_{\mathscr{F}^{n-i}}\right), \quad 0 \leq i \leq n .
\end{aligned}
$$

It is called the $\mathcal{F}$-cotriple resolution of $(R, A, \rho)$. Applying the functor $\mathcal{X} \mathrm{Ab}^{\text {add }}$ dimension-wise to $\mathscr{F}_{*}(R, A, \rho)$ we obtain the simplicial object $\mathcal{X} \mathrm{Ab}^{\text {add }} \mathscr{F}_{*}(R, A, \rho)$ in the category $X$ Vect.

Definition 4.5. The $n$-th cotriple cyclic homology of a crossed module of algebras $(R, A, \rho)$ is defined by

$$
\mathscr{H}_{n}(R, A, \rho)=H_{n}\left(\mathcal{X A b}^{\text {add }}\left(\mathcal{F}_{*}(R, A, \rho)\right)\right), \quad n \geq 0 .
$$

It is clear that $\mathscr{H}_{n}, n \geq 0$, is a functor from $\mathcal{X}$ Alg to $\mathcal{X}$ Vect. Moreover, for any $(R, A, \rho) \in \mathcal{X}$ Alg,

$$
\mathscr{H} \mathcal{C}_{0}(R, A, \rho) \cong \mathcal{X} \mathrm{Ab}^{\text {add }}(R, A, \rho)=(R /[A, R], A /[A, A], \bar{\rho}) .
$$

For further investigation of the cotriple cyclic homology of crossed modules of algebras we need some non-standard simplicial resolutions in the sense of Barr-Beck [1]. 
Proposition 4.6. Let $\left(\left(R_{*}, A_{*}, \rho_{*}\right),\left(d_{0}^{0}, d_{0}^{0}\right),(R, A, \rho)\right)$ be an augmented simplicial crossed module of algebras. Suppose the following conditions hold:

(i) the crossed module $\left(R_{n}, A_{n}, \rho_{n}\right), n \geq 0$, belongs to the projective class $\mathcal{P}$;

(ii) the augmented simplicial algebras $\left(R_{*}, d_{0}^{0}, R\right)$ and $\left(A_{*}, d_{0}^{0}, A\right)$ are aspherical. Then the simplicial crossed modules of algebras $\left(R_{*}, A_{*}, \rho_{*}\right)$ and $\mathcal{F}_{*}(R, A, \rho)$ are homotopically equivalent.

Proof. Straightforward from [1], 5.3.

Now we describe several connections between the cyclic homology of algebras and cotriple cyclic homology of crossed modules. There are two ways of regarding an algebra $A$ as a crossed module, via the trivial map 0:0 $\rightarrow A$ and via the identity map $1_{A}: A \rightarrow A$ with action of $A$ on itself given by multiplication. Respectively there are full embeddings

$$
i, \epsilon: \mathrm{Alg} \rightarrow X \mathrm{Alg}
$$

defined by $i A=(0, A, 0)$ and $\epsilon A=\left(A, A, 1_{A}\right)$. The functor $i$ has a left adjoint $\tau: \mathcal{X A l g} \rightarrow \operatorname{Alg}, \tau(R, A, \rho)=$ Coker $\rho$ and also a right adjoint $\kappa: X \mathrm{Alg} \rightarrow \mathrm{Alg}$, $\kappa(R, A, \rho)=A$. On the other hand, the functor $\kappa$ and the functor $\xi: X \mathrm{Alg} \rightarrow \mathrm{Alg}$, $\xi(R, A, \rho)=R$ are left and right adjoint to the functor $\epsilon$, respectively.

If we regard $\mathcal{X}$ Vect as a subcategory of $\mathcal{X} \mathrm{Alg}$, then the cotriple cyclic homology $\mathscr{H C}_{n}(R, A, \rho)$ can be presented as a linear map $\xi \mathscr{H}^{\mathcal{C}_{n}}(R, A, \rho) \rightarrow \kappa \mathcal{H C}_{n}(R, A, \rho)$.

Proposition 4.7. (i) For any crossed module $(R, A, \rho)$ and $n \geq 0$,

$$
\kappa \mathcal{H}_{n}(R, A, \rho) \cong \mathrm{HC}_{n}(A) .
$$

(ii) For any algebra $A$ and $n \geq 0$,

$$
\mathscr{H}_{n}(i A) \cong i \operatorname{HC}_{n}(A) \text { and } \mathscr{H}_{n}(\epsilon A) \cong \epsilon \operatorname{HC}_{n}(A) .
$$

Proof. (i) By Proposition 4.6 the simplicial algebra $\kappa \mathcal{F}_{*}(R, A, \rho) \rightarrow A$ is a free simplicial resolution of $A$. Therefore

$$
\kappa \mathscr{H}^{\mathcal{C}_{n}}(R, A, \rho)=\kappa H_{n}\left(\mathcal{X} \mathrm{Ab}^{\text {add }}\left(\mathcal{F}_{*}(R, A, \rho)\right)\right)=H_{n}\left(\operatorname{Ab}^{\text {add }}\left(\kappa \mathcal{F}_{*}(R, A, \rho)\right)\right) .
$$

The assertion follows from [7], Theorem 1.1.

(ii) Let $\left(A_{*}, d_{0}^{0}, A\right)$ be a free simplicial resolution of an algebra $A$. It is routine to check that $i A_{n}$ and $\epsilon A_{n}$ belong to the projective class $\mathcal{P}$ for any $n \geq 0$. Then by Proposition 4.6 we have

$$
\begin{aligned}
\mathscr{H} \mathcal{C}_{n}(i A) & \cong H_{n}\left(X \mathrm{Ab}^{\mathrm{add}}\left(i A_{*}\right)\right)=H_{n}\left(i \mathrm{Ab}^{\mathrm{add}}\left(A_{*}\right)\right)=i H_{n}\left(\mathrm{Ab}^{\mathrm{add}}\left(A_{*}\right)\right) \\
& \cong i \mathrm{HC}_{n}(A)
\end{aligned}
$$

and

$$
\begin{aligned}
& \mathscr{H}_{n}(\epsilon A) \cong H_{n}\left(\mathcal{X \mathrm { Ab } ^ { \mathrm { add } }}\left(\epsilon A_{*}\right)\right)=H_{n}\left(\epsilon \mathrm{Ab}^{\mathrm{add}}\left(A_{*}\right)\right)=\epsilon H_{n}\left(\mathrm{Ab}^{\mathrm{add}}\left(A_{*}\right)\right) \\
& \cong \epsilon \mathrm{HC}_{n}(A) \text {. }
\end{aligned}
$$


Finally, in this subsection we calculate the cotriple cyclic homology of an inclusion crossed module of algebras.

Proposition 4.8. Let $(I, A$, inc) be an inclusion crossed module of algebras. Then there is an isomorphism

$$
\xi \mathscr{H}_{n}(I, A, \text { inc }) \cong \operatorname{HC}_{n}(A, I), \quad n \geq 0,
$$

where $\mathrm{HC}_{n}(A, I)$ denotes the $n$-th relative cyclic homology.

Proof. Let $\mathcal{F}_{*}=\mathscr{F}_{*}(I, A$, inc $) \rightarrow(I, A$, inc $)$ be the cotriple resolution of $(I, A$, inc $)$. Note that both $\kappa \mathcal{F}_{n}$ and $\kappa \mathcal{F}_{n} / \xi \mathcal{F}_{n}$ are free algebras for each $n \geq 0$. Moreover, $\xi \mathcal{F}_{*} \rightarrow$ $I$ and $\kappa \mathcal{F}_{*} \rightarrow A$ are aspherical augmented simplicial algebras and since $(I, A$, inc) is an inclusion crossed module, the augmented simplicial algebra $\kappa \mathcal{F}_{*} / \xi \mathcal{F}_{*} \rightarrow A / I$ is aspherical as well. We have the commutative diagram of complexes

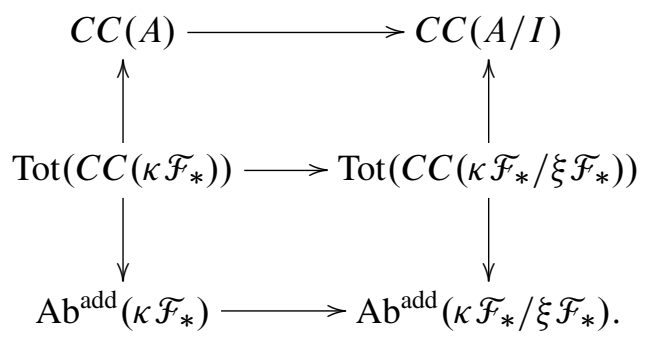

Clearly, by Lemma 2.3, the both vertical morphisms in the upper quadrant are quasiisomorphisms. Furthermore, by [7], the both vertical morphisms in the lower quadrant are also quasi-isomorphisms. Consequently, we have an isomorphism

$$
\mathrm{HC}_{n}(A, I) \cong H_{n}\left(\operatorname{Ker}\left\{\mathrm{Ab}^{\mathrm{add}}\left(\kappa \mathcal{F}_{*}\right) \rightarrow \operatorname{Ab}^{\text {add }}\left(\kappa \mathcal{F}_{*} / \xi \mathcal{F}_{*}\right)\right\}\right), \quad n \geq 0 .
$$

Now from the five-term exact cyclic homology sequence of [18] and the fact that $\mathrm{HC}_{1}\left(\kappa \mathcal{F}_{n} / \xi \mathcal{F}_{n}\right)=0, n \geq 0[16]$, we deduce

$$
\operatorname{Ker}\left\{\mathrm{Ab}^{\mathrm{add}}\left(\kappa \mathcal{F}_{*}\right) \rightarrow \mathrm{Ab}^{\mathrm{add}}\left(\kappa \mathcal{F}_{*} / \xi \mathcal{F}_{*}\right)\right\} \cong \xi \mathcal{F}_{*} /\left[\kappa \mathcal{F}_{*}, \xi \mathcal{F}_{*}\right] .
$$

But, by definition

$$
\xi \mathscr{H}_{n}(I, A, \text { inc })=\xi H_{n}\left(\mathcal{X} \mathrm{Ab}^{\text {add }} \widetilde{\mathcal{F}}_{*}\right)=H_{n}\left(\xi \mathcal{F}_{*} /\left[\kappa \mathcal{F}_{*}, \xi \mathcal{F}_{*}\right]\right) .
$$

This completes the proof.

Corollary 4.9. Let $(I, A$, inc) be an inclusion crossed module of algebras. Then there is a long exact homology sequence

$$
\begin{aligned}
\cdots & \rightarrow \xi \mathcal{H}_{n}(I, A, \text { inc }) \rightarrow \operatorname{HC}_{n}(A) \rightarrow \operatorname{HC}_{n}(A / I) \rightarrow \cdots \rightarrow \operatorname{HC}_{1}(A) \\
& \rightarrow \operatorname{HC}_{1}(A / I) \rightarrow I /[A, I] \rightarrow A /[A, A] \rightarrow A /(I+[A, A]) \rightarrow 0
\end{aligned}
$$

Proof. Straightforward from Proposition 4.8 . 


\section{Cyclic homology versus cotriple cyclic homology}

In this section we compare two cyclic homology theories of crossed modules of algebras discussed above. Namely, the aim of this section is to prove the following.

Theorem 5.1. Let $\mathbb{k}$ be a field of characteristic zero and $(R, A, \rho)$ a crossed module of algebras. Then there are natural exact sequences

$$
\begin{aligned}
\cdots & \rightarrow \operatorname{HC}_{n+1}(R, A, \rho) \rightarrow \xi \mathcal{H}_{n}(R, A, \rho) \rightarrow \operatorname{HC}_{n}(A) \rightarrow \operatorname{HC}_{n}(R, A, \rho) \\
& \rightarrow \xi \mathcal{H}_{n-1}(R, A, \rho) \rightarrow \cdots \rightarrow \xi \mathcal{H}_{1} \mathcal{C}_{1}(R, A, \rho) \rightarrow \operatorname{HC}_{1}(A) \\
& \rightarrow \mathrm{HC}_{1}(R, A, \rho) \rightarrow R /[A, R] \stackrel{\rho}{\rightarrow} A /[A, A] \rightarrow A /(\operatorname{Im} \rho+[A, A]) \rightarrow 0
\end{aligned}
$$

and

$$
\begin{aligned}
& \cdots \rightarrow \xi \mathscr{H} \bigodot_{n-1}(R, A, \rho) \rightarrow H_{n+1}(\beta(R, A, \rho)) \rightarrow \xi \mathcal{H} \complement_{n}(R, A, \rho) \\
& \rightarrow \xi \mathscr{H}^{\mathcal{C}_{n-2}}(R, A, \rho) \rightarrow H_{n}(\beta(R, A, \rho)) \rightarrow \cdots \rightarrow \xi \mathcal{H C}_{1}(R, A, \rho) \\
& \rightarrow H_{3}(\beta(R, A, \rho)) \rightarrow \xi \mathscr{H}^{C_{2}}(R, A, \rho) \rightarrow \xi \mathscr{H} \mathcal{C}_{0}(R, A, \rho) \\
& \rightarrow H_{2}(\beta(R, A, \rho)) \rightarrow \xi \mathscr{H}^{\varkappa_{1}}(R, A, \rho) \rightarrow 0 .
\end{aligned}
$$

Moreover, there are isomorphisms

$$
H_{1}(\beta(R, A, \rho)) \cong \xi \mathscr{H}_{0}(R, A, \rho) \cong R /[A, R],
$$

where the complex $\beta$ is defined immediately below in the next subsection.

Note that the sequence (15) coincides with relative cyclic homology exact sequence (14) when $(R, A, \rho)=(I, A$, inc), while the sequence (16) seems to be a generalisation of Connes' periodicity exact sequence.

5.2. The complexes beta and gamma. We need to define auxiliary complexes $\beta$ and $\gamma$. Given a crossed module $(R, A, \rho)$ of algebras, we have a natural morphism of crossed modules $\left(0,1_{A}\right):(0, A, 0) \rightarrow(R, A, \rho)$. It is easy to see that there are injective maps of bicomplexes

$$
C C^{\{2\}}\left(\Re_{*}\left(0,1_{A}\right)\right): C C^{\{2\}}\left(\Re_{*}(0, A, 0)\right) \rightarrow C C^{\{2\}}\left(\Re_{*}(R, A, \rho)\right)
$$

and

$$
C C\left(\Re_{*}\left(0,1_{A}\right)\right): C C\left(\Re_{*}(0, A, 0)\right) \rightarrow C C\left(\Re_{*}(R, A, \rho)\right),
$$

which yield the respective injective maps of complexes

$$
i_{(R, A, \rho)}: C C^{\{2\}}(0, A, 0) \rightarrow C C^{\{2\}}(R, A, \rho)
$$

and

$$
j_{(R, A, \rho)}: C C(0, A, 0) \rightarrow C C(R, A, \rho) .
$$


Define the complexes $\beta(R, A, \rho)$ and $\gamma(R, A, \rho)$ from the following commutative diagram of complexes of vector spaces with exact rows:

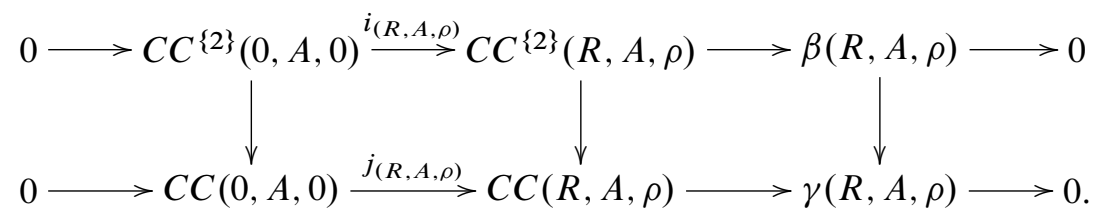

The next two propositions calculate the low dimensional homology of $\beta$ and $\gamma$ complexes.

Proposition 5.3. Let $(R, A, \rho)$ be a crossed module of algebras. Then we have

$$
H_{0} \beta(R, A, \rho)=H_{0} \gamma(R, A, \rho)=0
$$

and

$$
H_{1} \beta(R, A, \rho)=H_{1} \gamma(R, A, \rho) \cong R /[A, R]
$$

Proof. Given any simplicial algebra $A_{*}$, the last two rows of the bicomplexes $C C^{\{2\}}\left(A_{*}\right)$ and $C C\left(A_{*}\right)$ coincide. Namely, for the simplicial algebras $\mathfrak{N}_{*}(0, A, 0)$ and $\mathfrak{N}_{*}(R, A, \rho)$ they have the forms

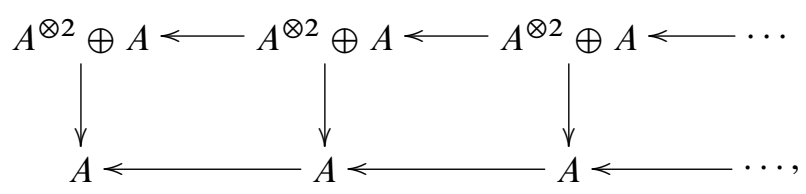

and

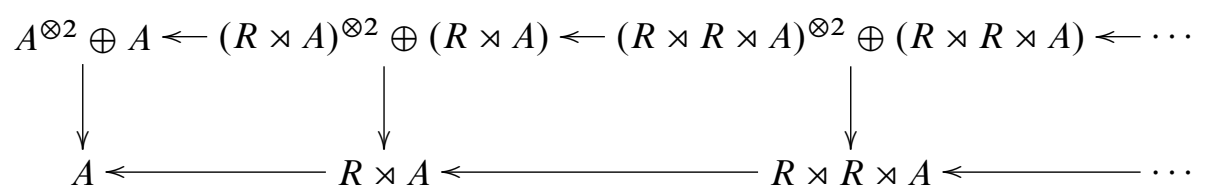

respectively. Hence, it is clear that $H_{0} \beta(R, A, \rho)=H_{0} \gamma(R, A, \rho)=0$. Moreover, comparing the given rows of the bicomplexes, we simply deduce that

$$
H_{1} \beta(R, A, \rho)=H_{1} \gamma(R, A, \rho)=\operatorname{Coker}\left((R \rtimes A)^{\otimes 2} / A^{\otimes 2} \rightarrow(R \rtimes A) / A\right),
$$

where the arrow is defined by

$$
(r, a) \otimes\left(r^{\prime}, a^{\prime}\right) \mapsto(r, a)\left(r^{\prime}, a^{\prime}\right)-\left(r^{\prime}, a^{\prime}\right)(r, a)=\left(\left[r, r^{\prime}\right]+\left[r, a^{\prime}\right]+\left[a, r^{\prime}\right],\left[a, a^{\prime}\right]\right) .
$$

This implies the isomorphism $H_{1} \beta(R, A, \rho)=H_{1} \gamma(R, A, \rho) \cong R /[A, R]$. 
Given an algebra $A$, by Corollary 2.4 the following pairs of complexes

$$
C C^{\{2\}}(0, A, 0), C C^{\{2\}}(A) \text { and } C C(0, A, 0), C C(A)
$$

are quasi-isomorphic. Then taking into account Proposition 5.3, for any crossed module of algebras $(R, A, \rho)$, the diagram (17) induces the morphism of long exact homology sequences

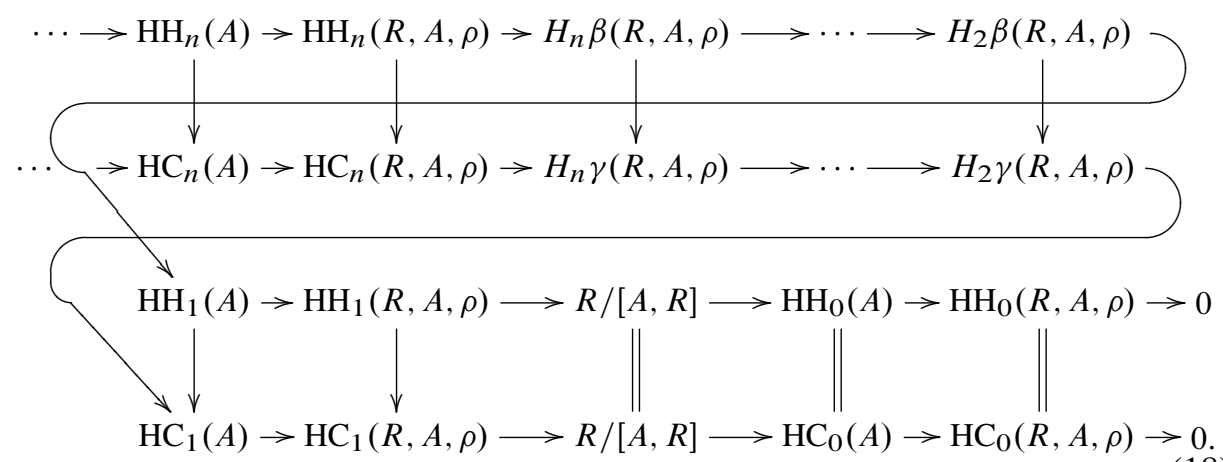

Proposition 5.4. Let $(R, A, \rho) \in \mathcal{X A l g}$ belong to the projective class $\mathcal{P}$ (see Subsection 4.4). Then

(i) $H_{n} \beta(R, A, \rho)=0$ for any $n>2$;

(ii) if in addition $\mathbb{k}$ is a field of characteristic zero, we have

$$
H_{n} \gamma(R, A, \rho)=0 \text { for } n>1
$$

and an isomorphism

$$
H_{2} \beta(R, A, \rho) \cong R /[A, R]
$$

Proof. Without loss of generality we can assume that $(R, A, \rho)=\mathbb{F}(V)$ for some $V \in$ Vect. Hence $R \stackrel{\rho}{\hookrightarrow} A$ is an inclusion crossed module with $A$ and $A / R$ being free algebras. Then, by Corollary 2.4 , the pairs of complexes $C C^{\{2\}}(R, A, \rho)$, $C C^{\{2\}}(A / R)$ and $C C(R, A, \rho), C C(A / R)$ are quasi-isomorphic.

Now (i) follows directly from the top exact sequence of the diagram (18) and the fact that, for a free algebra $F$, the Hochschild homology $\mathrm{HH}_{n}(F)$ vanishes for any $n \geq 2$.

If in addition $\mathbb{k}$ is a field of characteristic zero, then the cyclic homology $\operatorname{HC}_{n}(F)$ of a free algebra $F$ vanishes as well for any $n \geq 1$ (see [16], Proposition 5.4). Therefore the diagram (18) implies that $H_{n} \gamma(R, A, \rho)=0$ for $n>1$. Moreover, by Connes' periodicity exact sequence there is a natural isomorphism

$$
\mathrm{HC}_{0}(F) \stackrel{B}{\cong} \mathrm{HH}_{1}(F)
$$


Then the five term exact sequence in cyclic homology from [18] implies the commutative diagram with exact rows

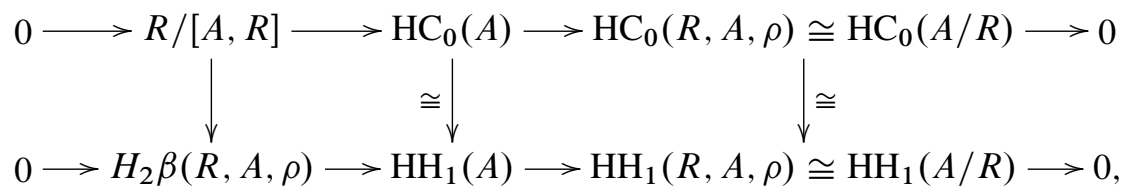

which completes the proof of (ii).

5.5. Proof of Theorem 5.1. Given a simplicial crossed module $\left(R_{*}, A_{*}, \rho_{*}\right)$ and a functor $\Psi: X \mathrm{Alg} \rightarrow \mathfrak{C}_{\geq 0}$, denote by $\Psi\left(R_{*}, A_{*}, \rho_{*}\right)$ the bicomplex of vector spaces obtained by applying the functor $\Psi$ dimension-wise to the simplicial crossed module $\left(R_{*}, A_{*}, \rho_{*}\right)$.

The following lemma will be needed.

Lemma 5.6. Let $\left(\left(R_{*}, A_{*}, \rho_{*}\right),\left(d_{0}^{0}, d_{0}^{0}\right),(R, A, \rho)\right)$ be an augmented simplicial crossed module of algebras. Suppose that $\left(R_{*}, d_{0}^{0}, R\right)$ and $\left(A_{*}, d_{0}^{0}, A\right)$ are aspherical augmented simplicial algebras. Then the augmented simplicial vector spaces

$$
\left(\beta_{n}\left(R_{*}, A_{*}, \rho_{*}\right), \beta_{n}\left(d_{0}^{0}, d_{0}^{0}\right), \beta_{n}(R, A, \rho)\right)
$$

and

$$
\left(\gamma_{n}\left(R_{*}, A_{*}, \rho_{*}\right), \gamma_{n}\left(d_{0}^{0}, d_{0}^{0}\right), \gamma_{n}(R, A, \rho)\right)
$$

are acyclic for any $n \geq 0$.

Proof. We shall prove only the acyclicity of the second augmented simplicial vector space. The proof of the first is similar and will be omitted.

Using the fact that the semidirect product of aspherical simplicial algebras is aspherical as well, the augmented simplicial algebra

$$
\left(\Re_{q}\left(R_{*}, A_{*}, \rho_{*}\right), \Re_{q}\left(d_{0}^{0}, d_{0}^{0}\right), \Re_{q}(R, A, \rho)\right), \quad q \geq 0,
$$

is aspherical. Hence, by Lemma 2.3 (i), we obtain that the augmented simplicial vector space

$$
\left(C C_{p}\left(\Re_{q}\left(R_{*}, A_{*}, \rho_{*}\right)\right), C C_{p}\left(\Re_{q}\left(d_{0}^{0}, d_{0}^{0}\right)\right), C C_{p}\left(\Re_{q}(R, A, \rho)\right)\right), \quad p \geq 0, q \geq 0,
$$

is acyclic. This clearly implies that $\left(C C_{n}\left(R_{*}, A_{*}, \rho_{*}\right), C C_{n}\left(d_{0}^{0}, d_{0}^{0}\right), C C_{n}(R, A, \rho)\right)$ and consequently $\left(C C_{n}\left(0, A_{*}, 0\right), C C_{n}\left(0, d_{0}^{0}\right), C C_{n}(0, A, 0)\right)$ are acyclic augmented simplicial vector spaces for any $n \geq 0$. Now the short exact sequence of augmented 
simplicial vector spaces

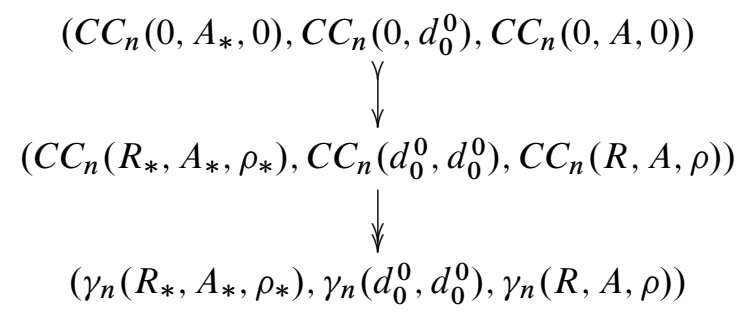

implies the result.

We return to the proof of Theorem 5.1.

Let $\left(\left(R_{*}, A_{*}, \rho_{*}\right),\left(d_{0}^{0}, d_{0}^{0}\right),(R, A, \rho)\right)$ be a simplicial resolution of $(R, A, \rho)$ in $X$ Alg in the sense of Barr-Beck (see Proposition 4.6) and let us consider the bicomplex $\gamma\left(R_{*}, A_{*}, \rho_{*}\right)$. For any fixed $q$ the homology of the complex $\gamma_{q}\left(R_{*}, A_{*}, \rho_{*}\right)$, by Lemma 5.6, is

$$
H_{p}\left(\gamma_{q}\left(R_{*}, A_{*}, \rho_{*}\right)\right) \cong \begin{cases}0 & \text { if } p>0 \\ \gamma_{q}(R, A, \rho) & \text { if } p=0\end{cases}
$$

Therefore $H_{n}\left(\operatorname{Tot}\left(\gamma\left(R_{*}, A_{*}, \rho_{*}\right)\right)\right) \cong H_{n}(\gamma(R, A, \rho))$. Hence there is a convergent spectral sequence

$$
E_{p q}^{1}=H_{q}\left(\gamma\left(R_{p}, A_{p}, \rho_{p}\right)\right) \Rightarrow H_{p+q}(\gamma(R, A, \rho)) .
$$

But by Propositions 5.3 and 5.4 we have

$$
E_{p q}^{1}= \begin{cases}0 & \text { for } p \geq 0, q \neq 1 \\ R_{p} /\left[A_{p}, R_{p}\right] & \text { for } p \geq 0, q=1\end{cases}
$$

Therefore $E_{p q}^{2} \cong \xi \mathcal{H}_{p}(R, A, \rho)$ for $q=1, p \geq 0$. Then the degenerate spectral sequence $E_{p q}^{2}$ yields the natural isomorphism

$$
H_{n+1}(\gamma(R, A, \rho)) \cong \xi \mathscr{H} \mathcal{C}_{n}(R, A, \rho), \quad n \geq 0 .
$$

Thus (18) and (19) imply the exact sequence (15). Furthermore, from (17) one easily deduces that for any crossed module $(R, A, \rho)$ there is a short exact sequence of complexes

$$
0 \rightarrow \beta(R, A, \rho) \rightarrow \gamma(R, A, \rho) \rightarrow \gamma(R, A, \rho)[2] \rightarrow 0
$$

where $\gamma(R, A, \rho)[2]$ is the dimension shifted complex by 2, i.e., $\gamma_{n}(R, A, \rho)[2]=$ $\gamma_{n-2}(R, A, \rho), n \geq 0$. Now the induced long exact homology sequence and the isomorphism (19) give the exact sequence (16). 


\section{References}

[1] M. Barr and J. Beck, Homology and standard constructions. In Seminar on triples and categorical homology theory (ETH, Zürich, 1966/67), Lecture Notes in Math. 80, SpringerVerlag, Berlin 1969, 245-335. Zbl 0176.29003 MR 0258917

[2] H.-J. Baues, Combinatorial homotopy and 4-dimensional complexes. With a preface by Ronald Brown, de Gruyter Exp. Math. 2, Walter de Gruyter \& Co., Berlin 1991. Zbl 0716.55001 MR 1096295

[3] H.-J. Baues and E. G. Minian, Crossed extensions of algebras and Hochschild cohomology. Homology Homotopy Appl. 4 (2002), 63-82. Zbl 1004.18012 MR 1918184

[4] R. Brown, Groupoids and crossed objects in algebraic topology. Homology Homotopy Appl. 1 (1999), 1-78. Zbl 0920.55002 MR 1691707

[5] P. Carrasco, A. M. Cegarra, and A. R.-Grandjeán, (Co)homology of crossed modules. J. Pure Appl. Algebra 168 (2002), 147-176. Zbl 0996.18010 MR 1887155

[6] P. Dedecker and A. S.-T. Lue, A nonabelian two-dimensional cohomology for associative algebras. Bull. Amer. Math. Soc. 72 (1966), 1044-1050. Zbl 0156.25903 MR 0210754

[7] G. Donadze, N. Inassaridze, and M. Ladra, Cyclic homology via derived functors. Homology, Homotopy Appl. 12 (2010), 321-334. Zbl 1236.16008 MR 2771592

[8] G. J. Ellis, Higher dimensional crossed modules of algebras. J. Pure Appl. Algebra 52 (1988), 277-282. Zbl 0658.17010 MR 952082

[9] G. J. Ellis, Homology of 2-types. J. London Math. Soc. (2) 46 (1992), 1-27; Corrigendum and addendum, ibid. 52 (1995), 447-448. Zbl 0713.18008 Zbl 0854.18008 MR 1180879 MR 1363812

[10] B. L. Fergin and B. L. Tsygan, Additive $K$-theory. In $K$-theory, arithmetic and geometry (Moscow, 1984-1986), Lecture Notes in Math. 1289, Springer-Verlag, Berlin 1987, 67-209. Zbl 0635.18008 MR 923136

[11] A.R.-Grandjeán, M. Ladra and T. Pirashvili, CCG-homology of crossed modules via classifying spaces. J. Algebra 229 (2000), 660-665. Zbl 0958.55007 MR 1769293

[12] H. Inassaridze, Non-abelian homological algebra and its applications. Math. Appl. 421, Kluwer Academic Publishers, Dordrecht 1997. Zbl 0936.18001 MR 1489738

[13] N. Inassaridze and M. Ladra, Hopf type formulas for cyclic homology. C. R. Math. Acad. Sci. Paris 346 (2008), 385-390. Zbl 1163.18005 MR 2417555

[14] C. Kassel and J.-L. Loday, Extensions centrales d'algèbres de Lie. Ann. Inst. Fourier (Grenoble) 32 (1982), 119-142. Zbl 0485.17006 MR 694130

[15] J.-L. Loday, Cyclic homology. 2nd ed., Grundlehren Math. Wiss. 301, Springer-Verlag, Berlin 1998. Zbl 0885.18007 MR 1600246

[16] J.-L. Loday and D. Quillen, Cyclic homology and the Lie algebra homology of matrices. Comment. Math. Helv. 59 (1984), 569-591. Zbl 0565.17006 MR 780077

[17] A. S.-T. Lue, Non-abelian cohomology of associative algebras. Quart. J. Math. Oxford Ser. (2) 19 (1968), 159-180. Zbl 0185.09304 MR 0248199

[18] D. Quillen, Cyclic cohomology and algebra extensions. K-Theory 3 (1989), 205-246. Zbl 0696.16021 MR 1040400 
[19] J. H. C. Whitehead, Combinatorial homotopy. II. Bull. Amer. Math. Soc. 55 (1949), 453-496. Zbl 0040.38801 MR 0030760

[20] M. Wodzicki, Excision in cyclic homology and in rational algebraic $K$-theory. Ann. of Math. (2) 129 (1989), 591-639. Zbl 0689.16013 MR 997314

Received October 20, 2010

G. Donadze, Department of Algebra, A. Razmadze Mathematical Institute and Tbilisi Centre for Mathematical Sciences, University Str. 2, 0186 Tbilisi, Georgia

E-mail: gdonad@gmail.com

N. Inassaridze, E. Khmaladze, Department of Algebra, A. Razmadze Mathematical Institute and Tbilisi Centre for Mathematical Sciences, University Str. 2, 0186 Tbilisi, Georgia, and Departamento de Matemática Aplicada I, Universidad de Vigo, E. E. Forestal, 36005 Pontevedra, Spain

E-mail: niko.inas@gmail.com; e.khmal@gmail.com

M. Ladra, Departamento de Álgebra, Universidad de Santiago de Compostela, 15782 Santiago de Compostela, Spain

E-mail: manuel.ladra@usc.es 